\title{
Convergence of basis expansions
}

\author{
Michael Blaskiewicz* \\ AGS Department Brookhaven National Laboratory
}

\begin{abstract}
An exactly solvable model of longitudinal bunched beam stability is used to test the convergence of the basis expansion formalism. It is found that basis expansions can predict instability for equations that have no unstable solutions.
\end{abstract}

\section{Introduction}

The basis expansion technique[1, 2, 3, 4,5] has been used for many years to find approximate eigenmodes in bunched beam instability problems. In general terms, one reduces the linearized Vlasov equation to an eigervalue problem in one spatial $(\tau)$ and one momentum $(v)$ variable,

$$
\lambda f(\tau, v)=L_{o p}[f(\tau, v)] .
$$

In equation (1) $\lambda$ is the eigenvalue, $f(\tau, v)$ is its eigenfunction, and $L_{o p}$ is a linear integro-differential operator. For all but the simplest cases $[6,7,8,9]$ no exact solutions are known. The basis expansion technique involves choosing a complete set of basis functions $g_{n}(\tau, v)$ and a weighting function $W(\tau, v)$ which satisfy an orthogonality relation

$$
\int g_{n}^{*}(\tau, v) g_{m}(\tau, v) W(\tau, v) d \tau d v=\delta_{m}^{n}
$$

In equation (2) the integral is over the domain where $W \neq 0$, the $*$ represents complex conjugate, the index $n$ represents an enumeration of the basis functions, and $\delta_{m}^{n}$ is the Kronecker delta. To proceed one uses completeness to write

$$
f(\tau, v)=\sum_{m=1}^{\infty} a_{m} g_{m}(\tau, v),
$$

\footnotetext{
*Work supported by the United States Department of Energy
} 
where the $a_{m} \mathrm{~s}$ are unknown coefficients.

One proceeds by multiplying equation (1) by $g_{n}^{*}(\tau, v) W(\tau, v) d \tau d v$ and integrates to obtain

$$
\lambda a_{n}=\sum_{m=1}^{\infty} T_{n, m} a_{m},
$$

where the matrix elements $T_{n, m}$ depend on the basis chosen, the impedance, etc. If $L_{o p}$ is sufficiently well behaved the technique seems to be exact to this point. In practical applications the infinite matrix equation is intractable, the sum is truncated at some value $N$, and eigenmodes are obtained using numerical techniques[3]. There is circumstantial evidence[9] that truncating the sum can lead to grossly incorrect eigenvalues even for large $N$.

The purpose of this paper is to examine the expansion technique using a very simple, exactly solvable model of bunched beam stability. The model is quite naive, but seems to incorporate the fundamental elements of a longitudinal instability calculation. It is found that one must be careful in drawing conclusions from the basis expansion results.

\section{Longitudinal instability model}

The model assumes a waterbag distribution in a square well longitudinal potential with an impedance $Z=R-i \omega L$. The particles undergo perfect reflection at the edges of the bunch which makes the rf restoring force a boundary condition[11, 12, $8,9,10]$.

Let $\theta$ denote machine azimuth, $\omega_{0}$ be the angular revolution frequency of a synchronous particle, and $\tau$ be arrival time relative to the head of the bunch. Using $\theta$ as the time-like variable the Vlasov equation away from the reflective boundaries is,

$$
\frac{\partial f}{\partial \theta}+v \frac{\partial f}{\partial \tau}-\kappa\left(I R+L \frac{\partial I}{\partial \tau}\right) \frac{\partial f}{\partial v}=0 .
$$

In equation (5): $f=f(\theta, \tau, v), I=I(\tau, \theta)=q \int d v f(\theta, \tau, v), v=d \tau / d \theta$, and

$$
\kappa=\frac{\eta}{2 \pi \omega_{0} \beta^{2}\left(E_{0} / q\right)},
$$

with slip factor $\eta$. The synchronous particle's energy, charge, and velocity are $E_{0}, q$, and $\beta c$, respectively. For a waterbag distribution below the wave-breaking threshold the solution to the Vlasov equation is of the form:

$$
f(\theta, \tau, v)=f_{0} H\left(v_{+}(\theta, \tau)-v\right) H\left(v-v_{-}(\theta, \tau)\right) H(\tau) H\left(\tau_{b}-\tau\right)
$$


where $f_{0}$ is a constant and $H(x)$ is the heaviside function with

$$
H(x)= \begin{cases}1, & \text { if } x>0 \\ 0, & \text { otherwise }\end{cases}
$$

Substituting equation (6) into (5) results in differential equations for $v_{+}$and $v_{-}$,

$$
\frac{\partial v_{ \pm}}{\partial \theta}+v_{ \pm} \frac{\partial v_{ \pm}}{\partial \tau}=F
$$

with

$$
F=-\kappa\left(I R+L \frac{\partial I}{\partial \tau}\right)
$$

For perfect reflection at the $\tau$ boundaries $v_{+}(\theta, 0)+v_{-}(\theta, 0)=0$ and $v_{+}\left(\theta, \tau_{b}\right)+$ $v_{-}\left(\theta, \tau_{b}\right)=0$. The current is proportional to $v_{+}-v_{-}$and the solution is exact to this point.

To obtain an exactly solvable model neglect the effect of $R$ on the unperturbed distribution setting

$$
v_{ \pm}= \pm \hat{v}+\delta v_{ \pm}(\tau) \exp \left(-i Q^{\theta}\right) .
$$

Setting $\delta v_{+}(\tau)+\delta v_{-}(\tau)=D(\tau)$ and keeping first order terms yields a single equation for $D$ with

$$
Q^{2} D=-\hat{v}(\hat{v}-2 V) \frac{d^{2} D}{d \tau^{2}}+2 U \hat{\vartheta} \frac{d D}{d \tau},
$$

where $U-i V=-q \kappa \omega_{0} f_{0}\left(R-i \omega_{0} L\right)$. For perfect reflection at $\tau=0$ and $\tau=\tau_{b}$ the boundary conditions are $D(0)=D\left(\tau_{b}\right)=0$. To solve (8) notice that

$$
D(\tau)=\exp \left(\lambda_{+} \tau\right)-\exp \left(\lambda_{-} \tau\right) .
$$

Inserting this expression yields a quadratic equation for $\lambda_{ \pm}$,

$$
\lambda_{ \pm}=\frac{U}{\hat{v}-2 V} \pm \sqrt{\frac{U^{2}}{(\hat{v}-2 V)^{2}}-\frac{Q^{2}}{\hat{v}(\hat{v}-2 V)}} .
$$

The boundary condition at $\tau_{b}$ gives $\lambda_{+}-\lambda_{-}=2 \pi i k / \tau_{b}$ with $k \neq 0$ an integer. The eigenvalue satisfies

$$
Q_{k}^{2}=\hat{v}(\hat{v}-2 V)\left\{\frac{k^{2} \pi^{2}}{\tau_{b}^{2}}+\frac{U^{2}}{(\hat{v}-2 V)^{2}}\right\}
$$

and the eigenvector is

$$
D_{k}(\tau)=\sin \left(k \pi \tau / \tau_{b}\right) \exp \left(\tau \frac{U}{\hat{v}-2 V}\right) .
$$


The functions $D_{k}$ for $k=1,2, \ldots$ form a complete set on $(0, \pi)$, so no eigenmodes have been missed. The right hand side of (9) is positive as long as $\hat{v}>2 \mathrm{~V}$, so resistance alone cannot cause instability. Additionally, reactance alone cannot cause instability, since no unperturbed distribution exists unless $\hat{v}>4 V$. To prove this consider the single particle Hamilonian which is given by

$$
H=v^{2} / 2+\kappa L I+\text { boundary terms. }
$$

For a stable unperturbed distribution the net pressure from the boundaries must be confining or zero. In particular $H \geq 0$ for $v=\hat{v}$. For $H=0, \hat{v}^{2}=-2 \kappa L I=4 \hat{v} V$, which implies $\hat{v}>2 V$ and a stable system. This is in agreement with [7] which considers the case of a pure inductance with a parabolic line density confined by a linear rf force. For such a system any self consistent unperturbed distribution is stable.

While the reader may disagree with the physical behavior, the fact that equations (9) and (10) represent the complete solution of (8) is inescapable. In fact, everything before equation (8) may be viewed as purely motivational without affecting the main results.

\section{Testing basis expansions}

Consider equation (8) with $\hat{v}(\hat{v}-2 V)>0$. Let $x=\pi \tau / \tau_{b}, \nu^{2}=Q^{2} \tau_{b}^{2} / \pi^{2} \hat{v}(\hat{v}-2 V)$, and $\tilde{R}=U \tau_{b} / \pi(\hat{v}-2 V)$. The equation becomes

$$
\nu^{2} D=-\frac{d^{2} D}{d x^{2}}+2 \tilde{R} \frac{d D}{d x}
$$

with boundary conditions $D(0)=D(\pi)=0$. In these variables the exact eigenvectors and eigenvalues are

$$
\begin{aligned}
D_{k}(x) & =\sin (k x) \exp (\tilde{R} x) \\
\nu_{k}^{2} & =k^{2}+\tilde{R}^{2} .
\end{aligned}
$$

Let the expansion functions be $g_{n}(x)=\sin (n x)$ with $W=2 / \pi$ for $0<x<\pi$. The eigenvalue in equation (4) is $\lambda=Q^{2}$ and the matrix element is

$$
T_{n, m}=n m \delta_{m}^{n}+\frac{8 \tilde{R} n m}{\pi\left(n^{2}-m^{2}\right)} \operatorname{odd}(n-m),
$$

where $\operatorname{odd}(n-m)=1$ if $n-m$ is odd, and $\operatorname{odd}(n-m)=0$ otherwise. In particular, when $n=m$ the term proportional to the reisistance is zero and there is no frequency shift in the weak coupling limit. This is consistent with the exact solution since the tune shift is $O\left(\tilde{R}^{2}\right)$. 
A computer code was used to find solutions to

$$
Q^{2} a_{n}=\sum_{m=1}^{N} T_{n, m} a_{m},
$$

for various values of $N$ and $\tilde{R}$. The only sophisticated part of the code is the eigenvalue solver[13] which is a well tested standard routine. Figure 1 shows the values of $Q$ as a function $\tilde{R}$ for $N=2$ and predicts instability for $\tilde{R} \gtrsim 1$. Setting $N=9$ gives Figure 2. The lowest frequency mode in Figure 2 is stable, and appears to be the case for all odd $N$. Also notice that large values of $Q$ go unstable first. This also seems to be generic. Setting $\tilde{R} \rightarrow-\tilde{R}$ has no effect on the eigenvalue spectrum.

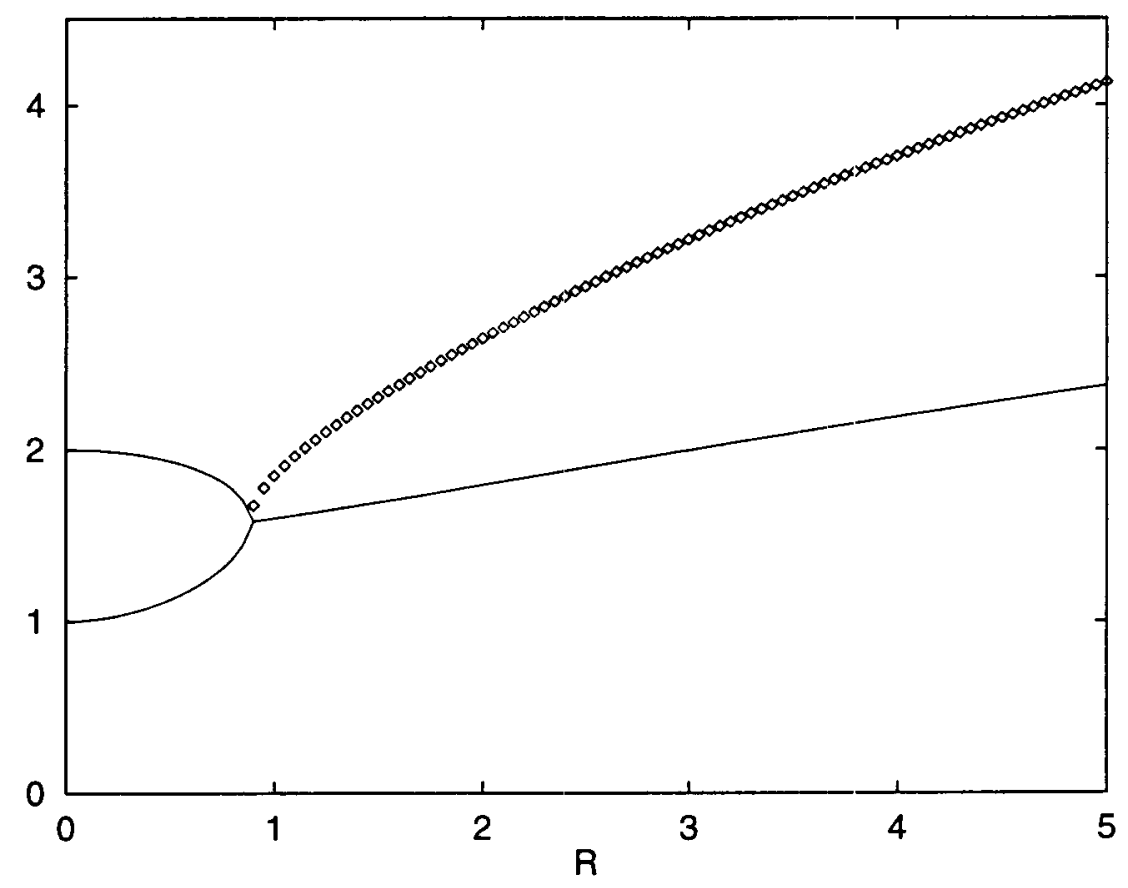

Figure 1: Real and imaginary parts of $Q$ versus $\tilde{R}$ for $N=2$. The solid lines are the real part of $Q$ and the distance between the points and solid line is the imaginary part of $Q$ for the unstable mode. 




Figure 2: Real and imaginary parts of $Q$ versus $\tilde{R}$ for $N=9$. The solid lines are the real part of $Q$ and the distance between the points and solid line is the imaginary part of $Q$ for the unstable mode.

As the number of modes increases the threshold value of $\tilde{R}$ for coupling between the lowest order modes continues to increase. Figure 3 shows the lowest 10 modes for $N=100$. The expansion technique predicts that lowest order modes go unstable for $\tilde{R}<5$, while the exact solution proves that the system is absolutely stable. Additionally, the threshold value of $\tilde{R}$ for the onset of unstable behavior without regard to mode varies only slightly with $N$. The threshold values of $\tilde{R}$ are 0.90 , 0.95 , and 0.95 for $N=2,9$, and 100 , respectively. With basis expansions alone could one show that equation (11) has no unstable solutions?

At the workshop it was suggested that the lack of convergence in the basis expansion technique might be due to the wide bandwidth of the model impedance. To test this hypothesis equation (11) is modified to read

$$
\nu^{2} D=-\frac{d^{2} D}{d x^{2}}+2 \tilde{R} \int_{0}^{\pi} S\left(x-x^{\prime}\right) d x^{\prime} \frac{d D\left(x^{\prime}\right)}{d x^{\prime}},
$$

where $S(x)$ is a smoothing function. Since convolution commutes with differentiation this is completely equivalent to assuming a smoothed wake potential instead of a delta funtion for the resistive term. To proceed consider the Fourier series expansion of the smoothing function

$$
S(x)=\sum_{n=0}^{\infty} S_{n} \cos (k x) .
$$


This function is periodic with $S(x)=S(x+2 \pi)$ but since the domain of $D(x)$ is $[0, \pi]$, which is half the period of $S(x)$, the value of the convolution within $[0, \pi]$ will exactly agree with the value obtained using the bounded support smoothing function $S_{b}(x)=H(x+\pi) H(\pi-x) S(x)$.

Inserting equation (17) into equation (16) and proceeding as before one obtains,

$$
T_{n, m}=n m \delta_{m}^{n}+\frac{4 \hat{R} n m}{\left(n^{2}-m^{2}\right)} \operatorname{odd}(n-m)\left(S_{n}+S_{m}\right) .
$$

For a delta function $S_{n}=1 / \pi$ for $n \geq 1$ and equation (18) reduces to equation (14). To test the effect of smoothing set $S_{n}=\exp \left(-\sigma^{2} n^{2} / 2\right) / \pi$ for $n \geq 1$. This is the periodic extension of a Gaussian with a root mean square width $\sigma$. Note that since $S_{0}$ does not enter equation (18) one is free to assume that $S(\pi)=0$. Figure 4 shows the lowest 10 modes for $N=100$ with $\sigma=0.1$. The threshold value of $\tilde{R}$ for the lowest order mode is reduced by smoothing the resistive term. However, the threshold value of $\tilde{R}$ without regard to mode is increased from 0.95 to 3.8 . Reducing $\sigma$ to 0.05 increases this to 4.75 . With $\sigma=0.02$ the threshold value of $\tilde{R}$ is 4.55 .

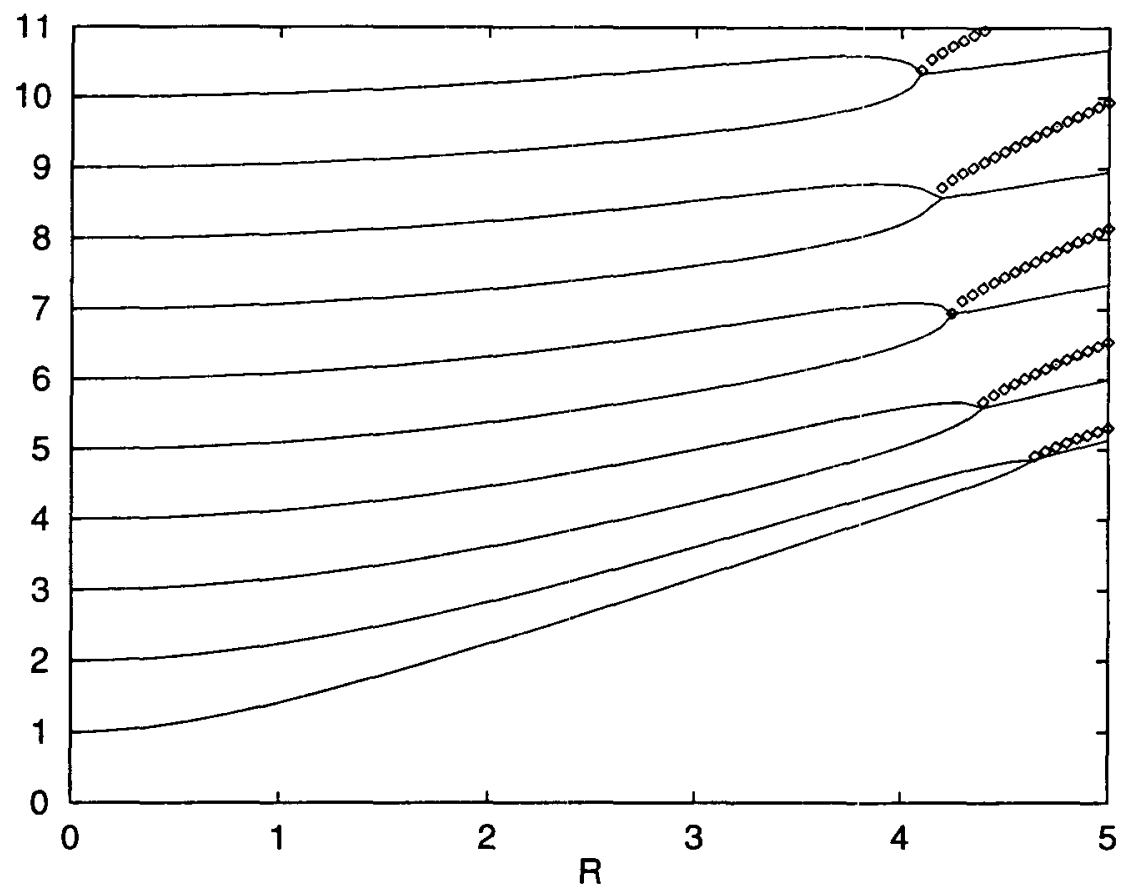

Figure 3: Lowest 10 eigenvalues versus $\tilde{R}$ for $N=100$. The solid lines are the real part of $Q$ and the distance between the points and solid line is the imaginary part of $Q$ for the unstable mode. 


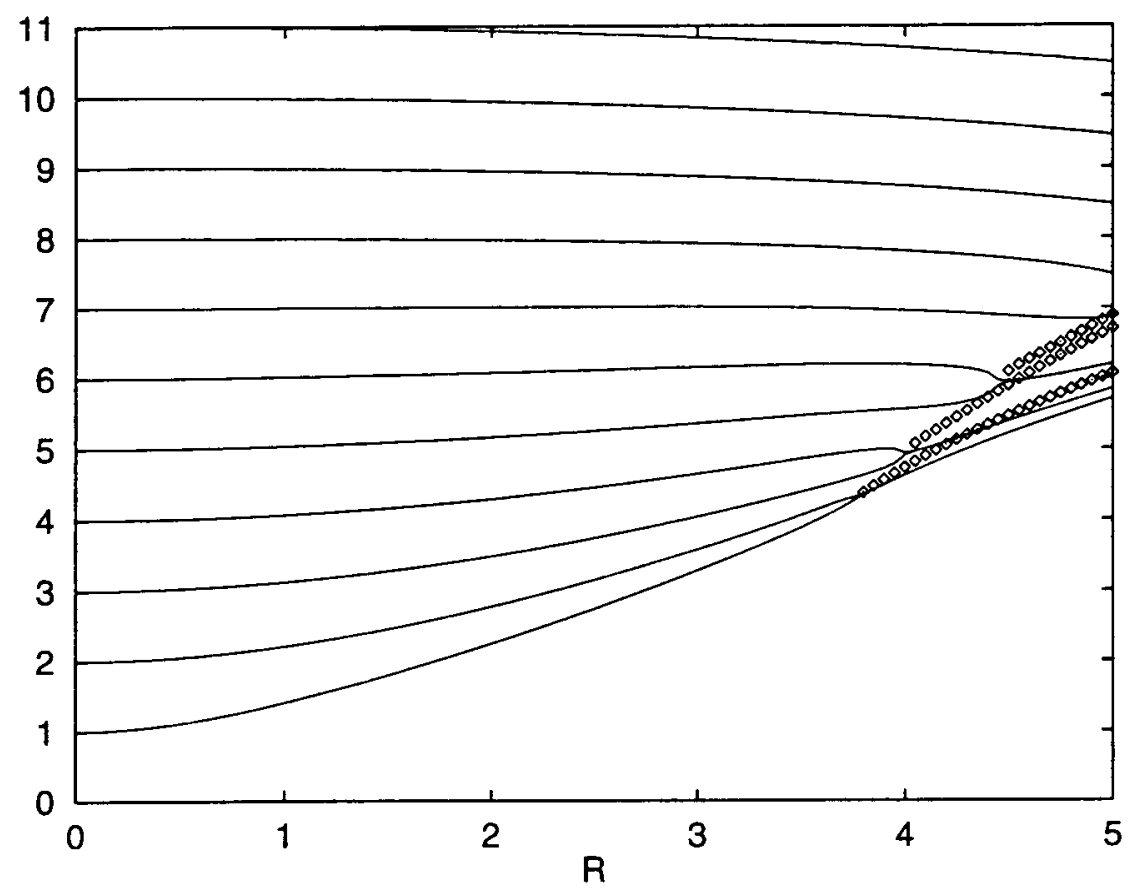

Figure 4: Lowest 11 eigenvalues versus $\tilde{R}$ using a Gaussian smoothing function for $N=100$ and $\sigma=0.1$. The solid lines are the real part of $Q$ and the distance between the points and solid line is the imaginary part of $Q$ for the unstable mode.

\section{Conclusions}

An exactly solvable model of longitudinal bunched beam stability including both resistance and inductance was presented and solved within the context of first order pertubation theory. It was found that the stability of the system depended only on the inductance. Including the effect of resistance on the unperturbed distribution (neglected here) may change this result. However, as discussed at the workshop, it may be that longitudinal microwave instability in bunched beams is an inherently nonlinear phenomena.

Regardless of the model's physical implications, the eigenvalue equation (11) seems quite reasonable and the basis expansion technique was applied to it. As is clear from Figures 1 through 3 the basis expansion technique predicts instability where none exists. Applying a spectral filter to the resistive term in the eigenvalue problem increased the stability threshold by as much as a factor of 4 . The general application of this technique requires a better understanding of the mathematics. 


\section{Acknowledgements}

My thanks to Alex Chao, Ingo Hofmann, Bruno Zotter and several others for interesting questions and illuminating discussions.

\section{References}

[1] K. Satoh, PEP Note 357 (1981).

[2] B. Zotter, CERN/SPS/81-20, (1981).

[3] Y.H. Chin, K. Satoh \& K. Yokoya, Particle Accelerators 13 , p45 (1983).

[4] G. Besnier, D. Brandt, B. Zotter, Particle Accelerators 17, 51-77, (1985).

[5] Y.H. Chin, CERN/SPS/85-9, (1985).

[6] F. Sacherer, CERN/SI-BR/72-5 (1972).

[7] D. Neuffer Particle Accelerators 11 p23 (1980).

[8] V.V. Danilov, E.A. Perevedentsev, 15th ICHEA p1163 (1992).

[9] M. Blaskiewicz, Phys Rev ST Accel Beams 1044201 (1998).

[10] M. Blaskiewicz, PAC99 TUP60 (1999).

[11] A.G. Ruggiero, BNL 51236, p 91. (1979)

[12] K.J. Kim, BNL 51236, p 100. (1979)

[13] The eispack routine cg, available through http://www.netlib.org 
